\title{
An Academic Model for Building Effective Faculty Teams to Promote Excellence in Nursing Education
}

\author{
Sally Cantwell”, Melissa Neville Norton, Valerie Gooder, Susan Thornock \\ Annie Taylor Dee School of Nursing, Weber State University, Ogden, USA \\ Email address: \\ sallycantwell@weber.edu (S. Cantwell),mneville@weber.edu (M. N. Norton), valeriegooder@weber.edu (V. Gooder), \\ sthornock@weber.edu (S. Thornock) \\ ${ }^{*}$ Corresponding author
}

\section{To cite this article:}

Sally Cantwell, Melissa Neville Norton, Valerie Gooder, Susan Thornock. An Academic Model for Building Effective Faculty Teams to Promote Excellence in Nursing Education. American Journal of Nursing Science. Vol. 9, No. 5, 2020, pp. 376-383.

doi: 10.11648/j.ajns.20200905.21

Received: September 24, 2020; Accepted: October 10, 2020; Published: October 16, 2020

\begin{abstract}
As a result of the national movement to advance nurses at the doctoral level, and the concurrent shortage of nursing faculty, the role of the DNP prepared nurse in the academic setting continues to evolve. At our School of Nursing, the need to define, understand and utilize the various faculty roles led to the development and implementation of an Academic Model for Nursing Education. This mixed-method research study was conducted to identify the factors that contribute to an effective culture in academia. The findings from the survey and focus groups indicated our model had a positive association on instructional design, teaching, administrative processes, adjustments of college tenure and promotion, clinical partnerships, shared faculty teaching loads, and succession planning. Focus group findings included the unanticipated benefits of improved faculty engagement, empowerment, collaboration, and faculty mentoring. Our academic practice model is reflective of the changes already occurring in collaborative clinical pathways in healthcare systems. The expected benefits of our academic practice model and those occurring in healthcare share comparable outcomes. These may include improving nursing scholarship, patient and student outcomes, strengthening the position of nursing in organizations and the broader community through enhanced leadership, providing parity with other healthcare disciplines, and improving the overall image of nursing [1]. Our Academic Model for Nursing Education is reflective of current healthcare practices, addresses the nurse faculty shortage while promoting faculty collaboration and job satisfaction.
\end{abstract}

Keywords: DNP-PhD Collaboration, Nursing Education, Faculty Teams, Academic Practice Model, Promotion, Outcomes, Scholarship

\section{Introduction}

The Health and Medicine Division (HMD) (formerly the Institute of Medicine), the Robert Wood Johnson Foundation, and The Joint Commission called for a transformation of nursing education [1-3]. The report on The Future of Nursing Leading Change, Advancing Health recommends substantial changes in nursing education, including increasing the number of doctorally prepared nurses. In response to the growing complexity of healthcare and concern surrounding quality and safety of patient care, the American Association of Colleges of Nursing (AACN) developed the practice doctorate as an alternative to the research-based doctorate. The introduction of DNP-prepared faculty to the academic environment is relatively recent but creates an excellent opportunity to advance nursing [4]. All faculty can work together; the Master's-prepared faculty using teaching expertise, the DNP-prepared faculty using their practice expertise, and the $\mathrm{Ph}$. D. -prepared faculty using their research experience [5]. In the clinical environment, these collaborations can improve patient outcomes [6].

As the number of DNP programs and graduates expands, the academic environment grows more diverse. Faculty roles continue to evolve in higher education. Faculty, regardless of degree type are sometimes unclear of their defined role. Role confusion between the DNP, Ed. D, and Ph. D. in research continues to exist. DNP-prepared faculty may feel marginalized and misunderstood regarding their abilities [7]. 
All doctoral degrees in nursing share more similarities than differences, the DNP, Ed. D, and Ph. D. are nurse leaders with scholarly backgrounds, who have accomplished terminal degrees that prepare them to perform in advanced nursing roles. Due to these similarities, along with their differences, the potential for strong collaborative teams on research and implementation of evidence-based practice benefits the healthcare system, academic setting, students, and patients.

The Carnegie Initiative on the Doctorate [8] suggested that additional attention is needed to promote a culture of intellectual discourse, participatory governance, openness, and fairness among all faculty members. Never has this been truer than now in nursing education. Changing roles and expectations may create some discomfort among all faculty in establishing the Carnegie Initiative. The ultimate vision is for all faculty, regardless of degree preparation, to partner in research, teaching, and implementation of evidence-based practice. Although there has been research done regarding the views of faculty related to the DNP role, there has not been a formal determination of the characteristics of the organizational culture required to support the MSN, DNP, $\mathrm{EdD}$, and $\mathrm{Ph}$. D. faculty model. In this research, we propose a model of an academic environment that promotes collaboration between all faculty. Our School of Nursing has employed DNP-prepared faculty since 2010, who have successfully participated in administration, teaching, shared governance, and collaborative research.

\section{Literature Review}

Over a decade ago, the American Association Colleges of Nursing (AACN) and their affiliate member schools, recommended the Doctor of Nursing Practice (DNP) as the new, terminal degree for advanced nursing practice [1]. This proposal represented a major professional paradigm shift in nursing practice and education that has resulted in a nationwide debate and divisiveness among stakeholders in healthcare and academia. Despite the growing numbers of DNP-prepared nurses, the impact of the DNP in the academic and hospital settings continues to be determined $[9,5]$. Dissension in the academic setting regarding the role of the DNP in nursing education continues [10].

At our institution, we have developed an organizational philosophy of engaging MSN-prepared, Ph. D. prepared, EdD-prepared, and DNP-prepared faculty in teaching, curriculum design, and collaborative research activities. These collaborative teams support the mission of our institution as well as the collaboration of faculty members. Research and anecdotal experiences indicate that collaborative faculty teams can provide nursing leadership in health care, bolster the equality of patient care, and improve patient outcomes [5].

The National League for Nursing (NLN) created a toolkit to support nurse leaders who intentionally want to promote and develop healthy work environments and facilitate faculty satisfaction. This toolkit provides many useful resources regarding the creation of safe, civil, and collegial nursing environments [11]. Our school of nursing has utilized many of the recommendations from the toolkit to build healthy faculty teams that has improved our academic culture. The NLN toolkit is recognized as a valid and reliable assessment tool; designed to focus faculty in evaluating nine areas which effect healthful work environments including salaries, benefits, workload, collegial environment, role preparation and professional development, scholarship, institutional support, marketing and recognition, and leadership. The NLN toolkit was developed to be used for measurement of job satisfaction, strategic planning and site-specific implementation [11]. The questions from the NLN toolkit were utilized as a guide for developing our research survey instrument for assessing organizational strengths and identifying limitations.

Building and maintain healthy academic work environments where nursing faculty can develop to attain their highest level of professional achievement is challenging [12]. Many new and senior nursing faculty report feeling disillusioned with their jobs as a result of lack of mentoring, orientation, minimal support from leadership, and the hierarchal environment. If left unaddressed, these stressors can result in decreased job satisfaction, role strain, and burnout. Academic leaders need to establish healthy work environments for nursing faculty for successful faculty recruitment, retention and collaboration in nursing education [12].

\section{Methods}

\subsection{Design}

The purpose of this mixed-method research study was to identify factors that contribute to an effective culture in academia; including the use of faculty teams on teaching, curriculum design, scholarship, tenure and promotion, and shared governance. A mixed-methods approach, including descriptive study design and focus group research, was used to achieve the study aims. The study and consent forms were evaluated by the Weber State University Institutional Review Board and approved with an exemption from full review.

\subsection{Sample and Recruitment}

Convenience sampling was used to recruit participants. Our school of nursing is part of a College of Health Professions. Faculty who were on the tenure and promotion track or who were already tenured, were recruited through email to participate in the survey questionnaire and focus groups. This convenience sample was recruited due to the faculty on tenure-track must show collaboration, engagement in scholarship, and service. Twenty-four faculty were eligible to participate. A follow-up email was sent to participants before closing the survey and as a reminder for voluntary focus group participation. Faculty responses were submitted to a built-in statistical software in Qualtrics ${ }^{\mathrm{XM}}$. All responses were coded without identifiers to protect respondent anonymity.

\subsection{Questionnaire}

Potential subjects were identified by the colleges' dean's office, who provided a list of nursing faculty who were 
tenured or on tenure and promotion track. Doctorally prepared and master's prepared faculty were included in the study. At our institution, master's prepared faculty can achieve tenure without a terminal degree and are full participants in teaching, scholarship, and service. As a preamble to the study, an introduction and rationale for conducting the survey, along with informed consent, was provided. Consent was assumed if the participant completed the anonymous survey. Survey questions were formulated by the research team using the reliable and validated National League for Nursing in their Healthful Work Environment Toolkit [11] as a guide.

Two researchers collaborated in adapting the survey using the NLN Toolkit. The questions were reviewed by the additional two researchers who reviewed the survey for content validity to ensure that the content domain was associated with survey constructs. The questionnaire was made available on a university-based online program to participants. The survey consisted of 18 questions (using a $7-$ point Likert scale) that ranged from 1-strongly agree to 7-strongly disagree, and demographics. The survey questions were analyzed using built-in statistical software in Qualtrics ${ }^{\mathrm{XM}}$ All responses were coded without identifiers to protect respondent confidentiality.

\subsection{Focus Groups}

Participants for the focus group were solicited by a separate informational email. Focus groups are a form of qualitative research, which includes a moderator who is impartial to the discussions [13]. Purposive sampling was utilized. All tenured faculty and those on tenure track and promotion at the school of nursing were invited to participate. All faculty were informed that participation was completely voluntary without it impacting their work or employment status. A non-nursing faculty member of the university's institutional effectiveness department with qualitative focus group training conducted the two focus group interviews. An overview of the study was provided and informed consent was obtained. There were nine faculty who participated in the focus groups. The focus group discussion was audiotaped and then transcribed by a third party. The transcriptions were given to the researchers for analysis, excluding any names or personal identifiers of participants.

Interpretive phenomenology guided the study design and analysis of the focus group transcripts and answers to the open-ended questions on the survey. Interpretive phenomenology is a design used to develop a thorough and thoughtful interpretation of the data while illuminating specific research questions [14]. A thematic analysis of the focus group transcripts and the open-ended questions in the survey were reviewed. Two investigators who did not attend the focus groups independently identified themes from the focus group interviews and then compared those themes to the open-ended survey responses. The investigators used field notes made by the focus group facilitator to provide additional context to the analysis. A third investigator independently participated in the same review process to ensure confirmability of the original findings. This was done to ensure consensus and to minimize the possibility of interpretation bias.

Table 1. Questions Included in the Online Survey.

\begin{tabular}{ll}
\hline Question Type & Questions Text \\
\hline 1. There is equitable treatment of faculty regardless of degree type in the School of Nursing. \\
2. Mentoring was available for the tenure and promotion process. \\
3. There is a sense of community and collegiality promoted in the School of Nursing. \\
4. I consider the work environment in the School of Nursing to be nurturing. \\
5. There are opportunities for advancement/promotion in the School of Nursing. \\
6. There are opportunities for participation in the Dumke College of Health Professions. \\
7. There are opportunities for participation at the university level at WSU. \\
8. Curriculum collaboration is valued in the School of Nursing. \\
9. Scholarly collaboration is valued in the School of Nursing. \\
10.I feel that my contributions matter in the School of Nursing. \\
11.I participate in shared governance in the School of Nursing. \\
12.I feel that I have the resources needed to grow in the profession. \\
13. There is a colleague in my department with whom I can ask for advice and guidance. \\
14.I feel like my "voice" is heard within the organization. \\
15.I participate in organizational decision-making. \\
16.I am given support to meet my professional goals. \\
17. Do nursing administrators and faculty share a common vision for the School of Nursing? \\
18. There is value shown for the unique contributions of all faculty members?
\end{tabular}

Table 2. Focus Group Questions.

\begin{tabular}{ll}
\hline Question Type & Questions Text \\
\hline & 1. Do you feel that all faculty are treated the same in the School of Nursing Why or why not? (Equity) \\
Focus Questions & 3. Describe the faculty mentoring process for tenure and promotion. (Academic Culture) \\
& 4. Would you consider the work environment in the School of Nursing nurturing? Examples? (Academic Culture) \\
& 5. Are there opportunities for advancement in the School of Nursing? (Academic Culture/Empowerment) Explain. \\
& 6. Are there opportunities for promotion in the School of Nursing? Explain (Academic Culture)
\end{tabular}




\begin{tabular}{l}
\hline Question Type \\
\hline 7uestions Text \\
8. Are there opportunities for participation at the College Level? Explain (Empowerment) \\
9. Is faculty collaboration valued in the School of Nursing? Explain. (Collaboration/Culture) \\
10. Is scholarly collaboration valued in the School of Nursing? Explain? (Collaboration/Culture) \\
11.Do you feel that your contributions are valued in the School of Nursing? Explain. \\
12. How do you participate in shared governance in the School of Nursing? (Academic Culture) Explain. \\
13.Do you have adequate resources needed to grow professionally? Please describe. Explain. (Empowerment) \\
14.Do you have access to colleagues in your department who you can ask for advice and guidance? (collaboration). Explain \\
15.Do you feel like your "voice" is heard within the organization? Explain. Empowerment \\
16. Do you have opportunities to participate in organizational decision-making at the School of Nursing? Explain. (empowerment) \\
17.Do you have opportunities to participate in organizational decision-making at the College level? Explain. \\
18.Do you have opportunities to participate in organizational decision-making at the university level? Explain.
\end{tabular}

\section{Results}

\subsection{Questionnaire}

Twenty of twenty-four faculty members completed the online survey for a response rate of $83 \%$ (Table 1). All participants had either a Ph. D., DNP, EdD, or MSN and were tenured or on the tenure and promotion track; of those participants, nine participated in the focus groups. Of the 20 who participated in the survey responses, two chose not to provide demographic information. Participant responses were anonymous and researchers had access to the grouped data but no individual responses.

Table 3. Faculty Survey Demographics.

\begin{tabular}{llll}
\hline Demographics & Ordinal Value & $\mathbf{\%}$ & $\mathbf{N}$ \\
\hline \multirow{4}{*}{ Age } & $20-30$ & 0 & 0 \\
& $31-40$ & $9.09 \%$ & 1 \\
& $41-50$ & $36.36 \%$ & 4 \\
& $51-60$ & $36.36 \%$ & 4 \\
& $61+$ & $18.18 \%$ & 2 \\
\hline
\end{tabular}

\begin{tabular}{llll}
\hline Demographics & Ordinal Value & $\mathbf{\%}$ & $\mathbf{N}$ \\
\hline & PhD & $27.78 \%$ & 5 \\
Educational Preparation & DNP & $5.56 \%$ & 1 \\
& EdD & $5.56 \%$ & 1 \\
& MSN & $62.12 \%$ & 11 \\
\hline & & & \\
\hline Demographics & Ordinal Value & $\mathbf{\%}$ & $\mathbf{N}$ \\
\hline \multirow{5}{*}{ Years in Academia } & $0-5$ years & $45.45 \%$ & 5 \\
& $6-10$ years & $27.27 \%$ & 3 \\
& $11-15$ years & $0.00 \%$ & 0 \\
& $16-20$ years & $18.18 \%$ & 2 \\
& $21-25$ years & $9.09 \%$ & 1 \\
& $25+$ years & $0.00 \%$ & 0 \\
\hline Demographics & & & \\
\hline \multirow{5}{*}{ Years Teaching at WSU } & Ordinal Value & $\mathbf{\%}$ & $\mathbf{N}$ \\
& $0-5$ years & $45.45 \%$ & 5 \\
& 6-10 years & $27.27 \%$ & 3 \\
& $11-15$ years & $0.00 \%$ & 0 \\
& $16-20$ years & $18.18 \%$ & 2 \\
& $21-25$ years & $9.09 \%$ & 1 \\
& $25+$ years & $0 \%$ & 0 \\
\hline
\end{tabular}

Table 4. Survey Responses.

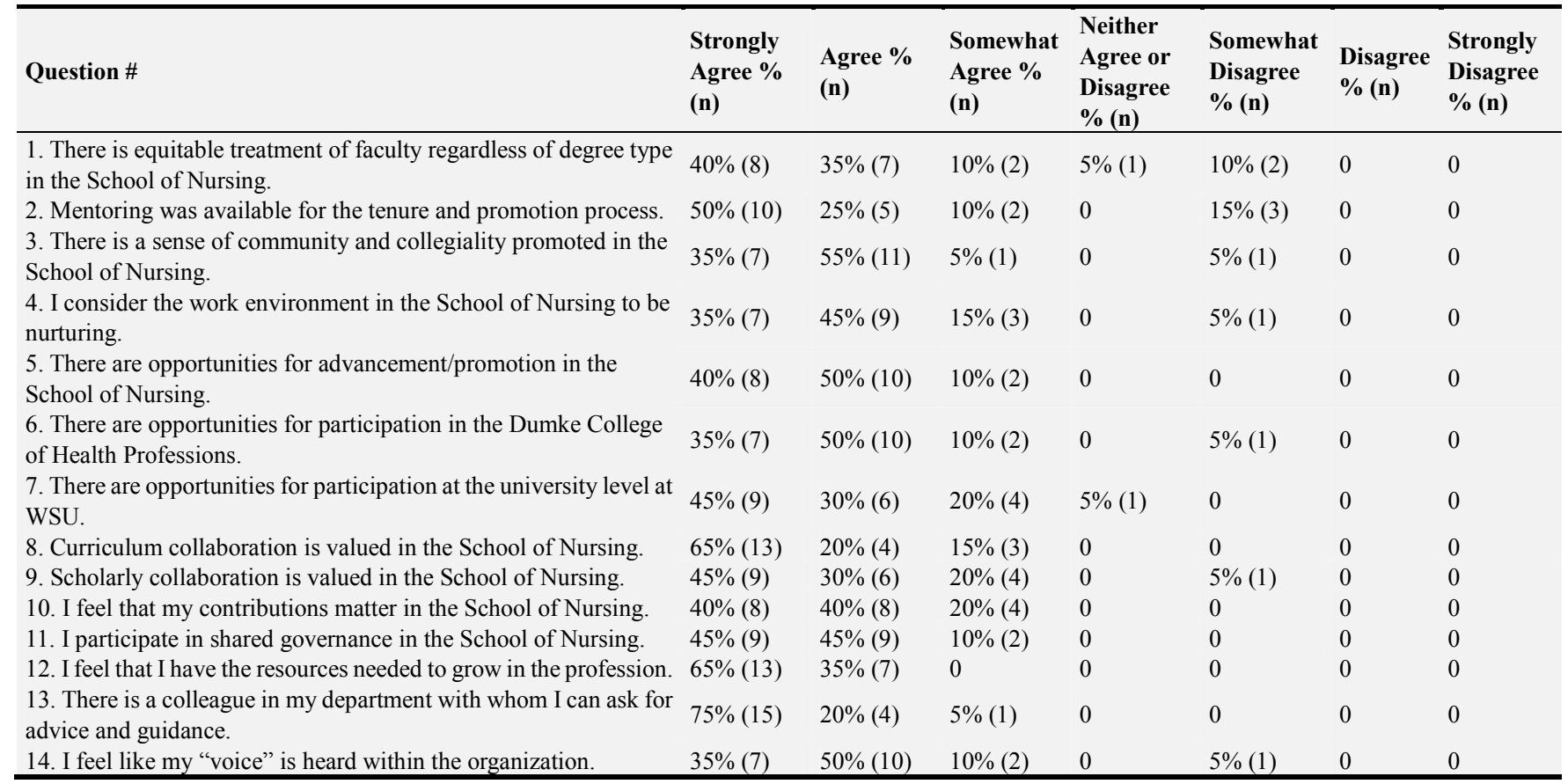




\begin{tabular}{|c|c|c|c|c|c|c|c|}
\hline Question \# & $\begin{array}{l}\text { Strongly } \\
\text { Agree \% } \\
\text { (n) }\end{array}$ & $\begin{array}{l}\text { Agree \% } \\
\text { (n) }\end{array}$ & $\begin{array}{l}\text { Somewhat } \\
\text { Agree \% } \\
\text { (n) }\end{array}$ & $\begin{array}{l}\text { Neither } \\
\text { Agree or } \\
\text { Disagree } \\
\% \text { (n) } \\
\end{array}$ & $\begin{array}{l}\text { Somewhat } \\
\text { Disagree } \\
\% \text { (n) }\end{array}$ & $\begin{array}{l}\text { Disagree } \\
\%(n)\end{array}$ & $\begin{array}{l}\text { Strongly } \\
\text { Disagree } \\
\%(n)\end{array}$ \\
\hline 15. I participate in organizational decision-making. & $40 \%(8)$ & $40 \%(8)$ & $15 \%(3)$ & 0 & 0 & $5 \%(1)$ & 0 \\
\hline 16. I am given support to meet my professional goals. & $70 \%(14)$ & $30 \%(6)$ & 0 & 0 & 0 & 0 & 0 \\
\hline $\begin{array}{l}\text { 17. Do nursing administrators and faculty share a common vision } \\
\text { for the School of Nursing? }\end{array}$ & $45 \%(9)$ & $35 \%(7)$ & $15 \%(3)$ & $5 \%(1)$ & 0 & 0 & 0 \\
\hline $\begin{array}{l}\text { 18. There is value shown for the unique contributions of all faculty } \\
\text { members? }\end{array}$ & $50 \%(10)$ & $10 \%(2)$ & $25 \%(5)$ & $5 \%(1)$ & $10 \%(2)$ & 0 & 0 \\
\hline
\end{tabular}

No demographic data was asked of the focus group participants. The inclusion data was communicated in the consent for focus group participation.

Two researchers independently analyzed the transcripts of the focus groups and the open-ended questions on the survey and identified the following themes.:

Academic Culture

Empowerment

Equity

Collaboration

Civility

Leadership

Through analysis of survey and focus group transcripts, academic culture emerged as the central theme and is identified as the domain of the Academic Nurse Educator Model. The remaining five themes indicate the influence on the domain and encompass a healthy work environment. See Figure 1. For the qualitative data, a coding process that involved researcher reflection was used as a way of interacting with and thinking about the data in a systematic manner [13]. Researchers worked systematically through the entire data set, giving attention to each data item from the survey responses and comparing them to the focus group findings, to identify commonalities that formed the basis of the themes. The final phase of the qualitative study involved sorting the relevant coded data into themes. Subthemes (inner circle, Figure 1) were identified as supportive topics for all main themes and emerged as shared topics upon analysis of the coding process. The combination of different data collection methods from the survey responses and focus groups was done to increase the probability that the research findings and interpretations were credible.

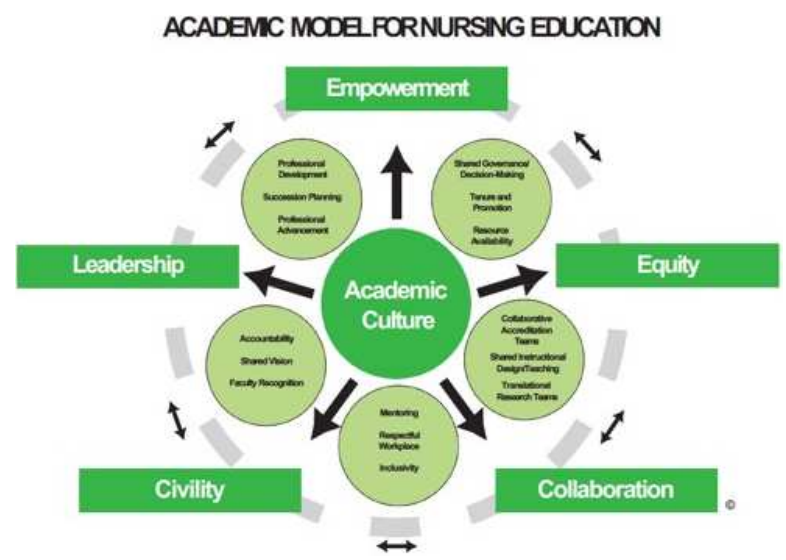

Figure 1. Academic Model for Nursing Education.

\subsection{Themes}

Academic Culture

Overall, the faculty agreed the academic culture included opportunities to be involved in decision-making and to be promoted. The majority of faculty $(n=19)(95 \%)$ share a common vision for the School of Nursing with administration. There were a small number of faculty who reported having a negative experience with mentoring upon hire however; all respondents $(n=20)(100 \%)$ report having a colleague they can go to for advice.

Do you have opportunities to participate in organizational decision-making at the School of Nursing?

"Maybe more than we want (laughter)."

Does the school of nursing respect all paths to the doctorate equally? Is there a perception that one doctoral pathway is better than another?

"We had a faculty who no longer works for us that did not. She was a person who felt that unless you had a Ph. D., you should not be tenured, not full-professor, and probably shouldn't even have been teaching in an academic environment. But again, that was one person."

"I think it depends on who you talk to. I really, truly believe, and I've had conversations with other faculty about all of this, it really depends on who you talk to, because I've talked to some that say that Ph. D. is the only way to go if you are going to be in education."

Did you feel that was of influence at the college or the SON, or indistinguishable?

"Definitely more so at the college level and I think those within the SON, again there wasn't enough information about the DNP or they didn't have the knowledge about it. But I felt like there was more support within the SON-not gung ho 'Yes we'll fully support this", but definitely not the resistance we felt from one particular faculty member, a very negative influence at the college."

You mentioned that was historically, what is the current sentiment you pick up?

"I've been here three years, and I've been asking a lot of people, the leaders in the SON, their opinions about what I should recommend education-wise. And what I've heard, I have non-biased information that a DNP is great, a Ph. D. is great - I was not led down a certain path at all." "It wasn't like that when I started. It was like, "your terminal degree is the Ph. D., you don't have an option for a DNP."

Let's talk about mentoring. How do you feel about the mentoring process in SON? Is it effective? Is it mentoring for new faculty? 
"I think what they are doing now is great - I'm on the faculty development committee - and I think what has been put in place is extremely valuable compared to what it was 5 or 6 years ago."

"Now, we have a whole website in place for people to refer to, new faculty are assigned a mentor that they work with enough through the first year, they are assigned -I know... has been a mentor; I've been a mentor three times and we are asked to meet with them weekly, you know, be available to ask, help them with any questions they may have in regards to courses or Canvas, or finding things on campus, whatever they may be. So, I feel like we have a really good system in place."

"I had an amazing mentor and so I had an amazing orientation process.

cross-teach across different programs levels, which I think is helpful because then you get to know other faculty that are teaching different things."

"I think our system is only as good as the mentor who is assigned to..."

That's true,

"and unfortunately people are very busy. In my experience, for instance, it was terrible. Any help that I got, I had to solicit myself. I cried every single day, [M] knows, and I said, 'I will quit when this semester is over.' Because I felt like nobody was there to help me. And I came from hospitals where you have this orientation and I was taught everything there and I wasn't taught anything here."

Would you consider the work environment in the School of Nursing nurturing?

"Very nurturing."

"And I think just from what I've seen and what I've heard from other people, we genuinely have a truly nurturing place compared to most."

Leadership

During the analysis of the open-ended survey questionnaire and the focus group scripts, leadership emerged as the central theme. Many of the Academic Culture topics reflected leadership principles.

Empowerment

All participants had a positive attitude toward feeling empowered. All faculty reported $(n=19)(100 \%)$ that they had the resources available to them to grow (one participant did not respond to this question); and all $(\mathrm{n}=20)(100 \%)$ reported that they felt support for meeting professional goals. In addition, a significant number of participants $(n=19)(95 \%)$ felt that their voice is heard within the organization.

Do you feel like your "voice" is heard within the organization?

"I think we are given plenty of opportunities for... and at least in the six years that I've been here, the 4 or 5 or 6 years that I've been here, there's been that call.

Do you have support for professional growth?

"Yes, I think so."

"I think when we have, when we go to conferences and interact with faculty from other universities, they are amazed at the financial support that we have."
Equity

Faculty feel they are valued and have equitable treatment regardless of degree type $(n=18) \quad(n=90 \%)$. Equal opportunities for participation in shared governance and decision-making was reported at $95 \%(n=19)$. All participants $(n=20)(100 \%)$ felt that their contributions to the school of nursing mattered.

Do you feel that all faculty are treated the same in the School of Nursing?

"I think it not necessarily related to the degree that I think we may be traded differently depending on our experience until we get to a certain point. I think it's good because we can't all be treated the same and when you first start, you don't have that much experience. You need a little bit more attention. You need to know some extra things when you've been here for a while. You need to be willing to think differently."

"they look at those individuals (faculty), individually and at their unique opportunity to contribute and then they decide how they might utilize the best."

Do you feel that your contributions are valued in the School of Nursing?

"Very much so."

Collaboration

All participants had a positive attitude regarding collaboration. A significant number of participants $(n=18)$ $(90 \%)$ felt that their collaborative contributions in curriculum were valued. Of those participating in scholarly collaboration $95 \%(n=19)$ felt it was valued. There are opportunities to work together and faculty expressed enjoyment in the cross-teaching approach and collaborative approach to accreditation preparation.

Do you feel respected by your faculty peers?

"I just think that the more that we collaborate and we get to know each other on a personal level, I think that makes a huge difference because then I can kind of get an insight into it; I think, ah, that's why they work the way they work."

Is faculty collaboration in general, valued at the school of nursing?

"I would say very much so."

"And one of the good things that we do is many of our faculty cross-teach across different program levels, which I think is helpful because then you get to know other faculty that are teaching different things. You can kind of align like what's happening amongst different programs."

"We just went through accreditation and we met as a faculty, um and, there were groups and sub-groups that were responsible for certain parts of that self-report and there was collaboration. But I think, bigger was, it was nice to get together as a group and discuss what the SON does, and that was a big collaborative effort. We always looked to leaders and people who had been around so it is, but that collaboration, that's at a high level. But we, most of us sit on committees where we evaluate curriculum, we weigh the outcomes of the program and we collaborate with faculty on courses and outcomes and everyone has the opportunity to come to the table. " 


\section{Civility}

Overall, faculty reported a civil work environment. There is some discussion regarding incivility between faculty but strong support of administration. All participants $(n=20)$ $(100 \%)$ reported having a sense of community and collegiality in the school of nursing and $(95 \%)(n=19)$ of participants consider the work environment to be nurturing.

Do you feel respected by your faculty peers?

"I feel respected by all the faculty."

"I've always been able to pop into whoever's office and ask questions. Everybody's very open and more than welcome to help."

"Something too is I've sat in a lots of like committee meetings and it's like totally safe to be like, no, I don't agree with you. I mean like you don't always get that in a lot of environments."

"I've been here long enough to know that that has changed and it is much better than it used to be."

What opportunities do you see for advancement in the SON?

"Either you get promoted based upon your tenure level from an assistant to associate. Or you move up on months of contract. And months of contract is generally associated with a director-type position. Nine-month versus 12."

So is there frustration about the lack of advancement opportunities (heads shaking), not necessarily.

"I think we are given plenty of opportunities for... and at least in the six years that I've been here, the 4 or 5 or 6 years that I've been here, there's been that call. That there's an administrative or leadership position that is available if you want to apply, so please let us know."

So, there's some opportunity on a fairly regular basis?

There's the opportunity to develop. We are a growing program

I have not seen those survey questions.

[c] Well - it was all about 'do your leaders support you?' But if you asked the same questions about faculty, it would have been different.

Okay, so you feel like you've got good faculty support, but not good leadership support?

[c] no, actually the opposite

[b], yes, the opposite.

\section{Discussion}

In the questionnaire, researchers examined the current state of civility, inclusivity, and academic collaborations in the School of Nursing. From the results of the survey responses, faculty reported a collaborative, inclusive work environment where they feel supported by administration and are provided with opportunities for advancement. Open communication, encouraged collaboration, and intentional teamwork are successful indicators and interventions used to improve an academic environment $[11,12]$. From the results of the survey responses, faculty reported a collaborative, inclusive work environment where they feel supported by administration and are provided with opportunities for advancement.
In the focus group, faculty perceptions and perspectives were more complex, but were consistent with the survey response findings. Focus group responses included various comments from faculty on poor to excellent mentoring experienced and highlighted recent improvements that had a significant impact on the faculty perspective of mentoring. The school of nursing has a standing, faculty-led development committee that focuses on a hybrid approach for a formal/informal mentoring program, with formal mentors assigned for the first 2 years of employment and ongoing informal mentoring with regards to course instruction and curriculum development, which has been evolving for the past four years. Faculty perspectives were consistent with a number of positive themes emerging, including mutual respect positive leadership, empowerment, and equity. These themes are consistent with the literature regarding determinants for successful workplace collaboration, team-based decision making, and a healthy academic culture [10,15,17]. Shared governance, team-based curriculum development, and a hybrid mentoring model are all interventions used to promoted collaboration. Intentional collaboration of faculty is viewed as one of the most successful tools in the overall healthy work environment and in the team approach [15]. Overall, the faculty who participated in the study reported an academic culture that promoted civility, inclusivity, and collaboration.

The researchers are employed at the university where the effective academic culture study was conducted, but did not participate in the anonymous survey or focus groups.

\section{Recommendations}

With an increasing number of DNP graduates anticipated and a predicted shortage of doctoral- prepared nurse faculty, faculty collaboration in scholarship, teaching, and practice must be established in the academic setting among all degree types $[16,17]$. The model for building effective faculty teams developed at our institution has been shown to increase employee satisfaction, faculty collaboration, and professional development opportunities. This collaborative model has given our students valuable clinical and leadership skills as well as research-focused training from combined faculty teams. The breadth of these teams has similarly contributed to student attainment of nursing competencies and student learning outcomes in our Associate, Baccalaureate, Master's, and Doctoral (DNP) programs.

\section{Conclusions}

At the foundation of our research findings, we discovered successful teams were centered around mutual respect and understanding of each other's roles. Identifying critical stakeholders within nursing as well as the college, allowed us to make changes to our bylaws and policies that defined academic qualifications, specifically tenure and promotion, and committees, which allowed us to introduce the role of the DNP as a qualified terminal degree. We found that the terminal degree is what qualified faculty to be hired into their 
position. Still, the academic efforts once employed, including curriculum development, committee work, scholarship, and teaching was equivalent, regardless of terminal degree. Providing opportunities for faculty to work together in teams, implementing combined mentoring models, and collaborative accreditation models were just a few interventions utilized in developing a successful DNP-PhD Nurse Educator Model. Collaborative relationships between faculty continue to evolve as the landscape of nursing education changes [18, 19]. The study findings emphasize the importance of communication, collaboration, equity, inclusivity, civility, and leadership as essential components to a healthy academic culture and reliable nurse faculty teams.

\section{References}

[1] American Association of Colleges of Nursing. (2004). AACN position statement on the practice doctorate in nursing. American Association of Colleges of Nursing (AACN). http://www.aacnnursing.org/Portals/42/DNP/DNPpositionstat ement.pdf?ver=2017-08-01095733-307.

[2] Institute of Medicine (IOM). (2011). The future of nursing: Leadingchange, http://doi.org/10/17226/12956 advancinghealth.

[3] The Joint Commission (2003). Strategies for addressing the evolving nursing crisis. JointCommission Journal on Quality and Safety, $29 \quad$ (1), https://doi.org/10.1016/S15493741(03)29006-7

[4] O'Connor, B. (2015). New White Paper on the Doctor of Nursing Practice: Current Issues and Clarifying Recommendations. Journal of Professional Nursing, 31 (5), 378. https://doi.org/10.1016/j.profnurs.2015.08.002

[5] Buchholz, S. W., Yingling, C., Jones, K., \& Tenfelde, S. (2015). $\mathrm{DNP}$ and PhD collaboration: Bringing together practice and research expertise as predegree and postdegree scholars. Nurse Educator, 40 (4), 203-206. https://doi.org/10.1097/NNE.0000000000000141

[6] Melnyk, B. M. (2013). Distinguishing the preparation and roles of doctor of philosophy anddoctor of nursing practice graduates: National implications for academic curricula and healthcare systems. Journal of Nursing Education, 52 (8), 442-448. https://doi.org/10.3928/0148483420130719-01

[7] Dreher, H. M., Smith Glasgow, M. E., Cornelius, F. H., \& Bhattacharya, A. (2012, December). A Report on a National Study of Doctoral Nursing Faculty. Nursing Clinics of North America, Vol. 47, pp. 435-453. https://doi.org/10.1016/j.cnur.2012.07.001095733-307.
[8] Walker, G. E. (2004). The Carnegie Initiative on the Doctorate. In D. H. Wulff \& A. E. Austin (Eds.), Paths to the professoriate: Strategies for enriching the preparation for future faculty. (pp. 236-249). Jossey-Bass.

[9] Tussing, T. Brinkman, B. Francis, D., Hixon, B., Labardee, R. Chipps, E. (2018). The impact of the doctorate of nursing practice nurse in a hospital setting. JONA: The Journal of Nursing Administration. 48 (12), 600-602. https://doi:10.1097/NNA.0000000000000688

[10] Staffileno, B., Pencak Murphy, M., \& Carlson, E. (2016). Determinants for effective collaboration among DNP-and PhD-prepared faculty. Nurse Outlook, 65 (1), 94-102. https://doi:10.1016/j.outlook.2016.08.003.

[11] National League for Nursing. (2018). Healthful work environment toolkit. Retrieved from: http://www.nln.org/docs/default-source/professional-develop ment-programs/healthful-work-environment-toolkit.pdf?sfvrsn $=20$

[12] Cooke, M. \& Valentine, N. M. (2020). Improving Teamwork and Communication in Schools of Nursing. Journal of Nursing Care Quality, 1-6 http://doi:10.1097/NCQ.0000000000000513.

[13] Cheng, K. W. (2014). A study on applying focus group interviews on education. Reading Improvement, 51 (4), 381-384.

[14] Brocki, J. M. \& Wearden, A. J. (2006). A critical evaluation of the use of interpretive phenomenologic analysis (IPA) in health psychology. Pyschology and Health, 21 (1), 87-108.

[15] Cowan, L., Hartjes, T. \& Munro, S. (2019). A model of successful DNP and $\mathrm{PhD}$ collaboration. Journal of the American Association of Nurse Practitioners, 31 (2), 116-123. https://doi:10.1097/JXX.0000000000000105.

[16] Falkenberg-Olson, A. C. (2019). Research translation and the evolving $\mathrm{PhD}$ and $\mathrm{DNP}$ practice roles. Journal of the American Association of Nurse Practitioners, 31 (8), 447-453. https://doi: 10.1097/JXX.0000000000000266.

[17] Tyczkowski, B. L. \& Reilly, J. (2017). DNP-Prepared nurse leaders: Part of the solution to the growing faculty shortage. JONA, $47 \quad(7 / 8), \quad 359-360$. https://doi:10.1097/NNA.0000000000000494

[18] Cygan, H. R., \& Reed, M. (2019). DNP and PhD scholarship: Making the case for collaboration. Journal of Professional Nursing, $\quad 35 \quad$ (5), 353-357. https://doi.org/10.1016/j.profnurs.2019.03.002

[19] Murphy M. P., Staffileno B. A., Carlson E. (2015). Collaboration among DNP and $\mathrm{PhD}$ prepared nurses: Opportunity to drive positive change. Journal of Professional Nursing, 31, 388-394. https://doi:10.1016/j.profnurs.2015.03.001. 